\title{
Human In Vitro Models of Ischaemic Stroke: a Test Bed for Translation
}

\author{
Ana Antonic • Emily S. Sena • Geoffrey A. Donnan • \\ David W. Howells
}

Received: 18 March 2012 /Revised: 27 June 2012 / Accepted: 12 July 2012 / Published online: 21 July 2012

(C) Springer Science+Business Media, LLC 2012

Our increased understanding of the ischaemic cascade has driven the rush to develop neuroprotective interventions. Over 500 have been reported to effectively improve outcome in experimental animal models of stroke. Although a number of well-conducted clinical trials have been performed, we have failed to reproduce these effects in humans [1]. Despite these difficulties in translation, neuroprotection remains an important potential therapy. If safe and easily administered, potentially in a pre-hospital setting, even small absolute benefits might have a significant impact.

Translational failure has been hotly debated, and many plausible explanations have been put forward. These include the possibility that clinical trials may have failed to detect neuroprotection where it does in fact exist, that animal studies may overstate the efficacy of neuroprotective interventions they test, and that the experimental models that we use may not replicate human stroke with sufficient fidelity. We do not believe that animal models are inherently faulty; indeed, the evidence suggests that the main themes of stroke biology hold true across all mammals studied so far. Clinical trial design and the strength and weaknesses of animal models and their employment are the subject of intense study [1-5], and in vivo testing will remain an important part of our overall armamentarium.

However, we need to consider the possibility that rodent molecular targets might not all be present in humans. Moreover, without robust target identification and proof-of-

\footnotetext{
A. Antonic · E. S. Sena · G. A. Donnan · D. W. Howells $(\square)$

The National Stroke Research Institute,

Florey Neuroscience Institutes,

245 Burgundy Street,

Heidelberg, Melbourne, Australia 3084

e-mail: david.howells@unimelb.edu.au

E. S. Sena

Centre for Clinical Brain Sciences, University of Edinburgh,

Edinburgh, UK
}

principal demonstration that attacking these targets elicits appropriate cellular responses, the foundations of our translational pyramid are weak and the pyramid itself is subject to collapse. Importantly, when the final target species is Homo sapiens, there is little logic to in vitro testing in cells from other species if human cell cultures are available at similar cost.

Why might humans and rodents not share the same molecular targets? While both species share all the hallmarks of mammals, they are separated by 80 million years of evolution [6]. Humans have large gyrencephalic brains with a high proportion of white matter, while most experimental animals have small smooth brains with relatively little white matter [7]. Within the cortex, the detailed architecture is different, with cross-species variations in functional maps and synaptic density [8]. Although we may share $90 \%$ of our genome with rodents [9] and have $93 \%$ homology with the rhesus macaque (Macaca mulatta) [10], a $10 \%$ difference implies that up to 3,000 genes may be different. Even those genes with homology may still have evolved different biochemistry and function. For example, mutations that cause ornithine transcarbamylase and phenylalanine hydroxylase deficiency in humans are present in the macaque genome, but are not associated with disease [10].

The recent disastrous clinical trial of TGN1412 that nearly killed six healthy volunteers provides another example of the risks of assuming molecular targets are identical across species. TGN1412 is believed to act through the CD28 receptor, of which the extracellular domain shares nearly $100 \%$ homology between humans and rhesus monkeys [11]. It is now believed that the binding characteristics of the two proteins differ and that this accounts for the severe inflammatory reactions seen in humans, but not in rhesus monkeys [12]. Excitotoxicity, one of the critical early events in the ischaemic cascade, differs between species. In mice, these events, as measured in the extracellular fluid of the 
ischaemic penumbra, may be over within $2 \mathrm{~h}$, while in human CSF, they may continue for 24h [13]. Human CSF turnover occurs approximately every $7 \mathrm{~h}$; thus, glutamate detected $24 \mathrm{~h}$ post-stroke is likely to be indicative of ongoing excitotoxicity in humans. Astrocyte function and particularly the pattern of glutamate transporter expression [14] may account for these potentially important differences. Since the concept of excitotoxicity is central to our understanding of the events which follow stroke, this may be a critical difference between the species. Developing a fast and effective human cell culture model of stroke may provide further insight into the efficacy of candidate interventions in the human brain and may help explain why many neuroprotective agents have failed in the clinic. A human in vitro system to test prospective neuroprotective agents is now a realistic way of bridging the gap between animal studies and clinical trials.

In the past, this has not been possible because of the lack of appropriate tools. In 2007, we performed a systematic search of the literature in PubMed using the search terms [ischaemia or stroke] and identified 200,000 studies. Of these, only 25 were identified by a sub-search on [human and in vitro]. In 2011, while an additional 100,000 studies on stroke have been reported, only five additional studies were performed in human in vitro systems. Of the 30 studies identified, 28 used immortalised cell lines derived from human neuroblastomas. These cells are not ideal models to study ischaemic stroke because of their ability to survive under hypoxic conditions and possession of abnormal karyotypes, which leads to constant cell division (not a feature of normal neurons) [15]. The remaining two studies took a more relevant but less practical approach of examining human slice preparations subject to oxygen-glucose deprivation. Both reported that human slices were more resistant to ischaemic insult than rat slices. Both examined excitotoxicity, one evaluated the activation of the NMDA receptor [16] and the other monitored the release of ${ }^{3} \mathrm{H}$-labelled D-aspartate efflux (a marker of newly taken up glutamate/aspartate, during and after superfusion with an ischaemic medium [17]). Neither study examined neuronal cell death as an outcome measure.

The use of human in vitro models of ischaemic stroke is rare. The method used to evaluate neuroprotective agents requires reform, and assessing drugs in human tissue may improve this process. To this end, it is important to develop appropriate human in vitro systems that model human disease with sufficient fidelity to be useful in translation. These models can be used to screen for relative activity. If the appropriate human in vitro models were used to evaluate the effects of TGN1412, the adverse effects associated with this drug would have been predicted. Testing of appropriately activated human T cells elicited the TGN1412-mediated cytokine release that put people's lives at risk [18]. Drug screening in characteristic myeloid, erythroid and megakaryocyte colonies derived from human embryonic stem cells [19] might have avoided this risk. With systemic and central inflammatory processes as a key facet of stroke biology and source of potential therapeutic targets, an ability to test for activity not only in neurons but also in a range of human tissues is important.

We propose that human embryonic stem cells (ESCs) could provide a source of unmodified, physiologically functional and mature human cells. Setting aside arguments on the ethics of their use, ESCs offer significant advantages over other human stem cell types with potential for use as screening tools. Adult human stem cell lines are currently less flexible in their use than ESC-derived lines. This lack of flexibility is a consequence of their partial pre-commitment to a particular differentiation pathway [20]. This makes expansion of numbers more difficult (a critical requirement for high-throughput screening) and requires greater complexity in the processes required for differentiation if multiple final cell types are required. Induced, pluripotent stem cells (iPSCs) have also yet to live up to their initial promise. Importantly, while iPSCs and ESCs have similar transcriptional profiles, the embryoid bodies derived from the two cell sources (and currently critical for differentiation) have very different transcriptional profiles [21]. Thus, while they may ultimately be important tools for exploring the impact of an individual's genetic background on risk of stroke and may allow personalised medicine in less time-constrained diseases, they offer little advantage as an initial screening tool. Conversely, ES cells can be maintained in culture for a large number of passages and can easily be differentiated towards a range of different cell types [15]. This is important because high-throughput screening will be most effective when all of the cell types which might contribute to a disease are available. For stroke, this would include not only neurons, astroglia, oligodendroglia and microglia but also vascular endothelial cells, pericytes, vascular smooth muscle cells and circulating inflammatory cells. Such cultures lend themselves to the detailed and systematic evaluation of drug-induced gene expression and cellular biochemistry under a range of physiological and injurious conditions $[22,23]$ and permit the use of relatively straightforward antisense and transfection technologies to modify the experimental environment.

When the final target species is Homo sapiens, there is little logic to in vitro testing in cells from other species if human cell cultures are available at similar cost. Since in vitro testing is considerably cheaper than in vivo testing, it is also the logical place to conduct candidate screening experiments. For example, in our own laboratory, it costs approximately $\$ 1,000$ to induce stroke in one rat and maintain it for $24 \mathrm{~h}$ of examining outcome with simple behavioural and histology testing. Therefore, to examine just a dose-response relationship with 
infarct volume as the outcome measure, it costs approximately $\$ 90,000$ for six drug concentrations with a cohort size of 15 powered to detect a $30 \%$ effect at the 0.05 level. To perform an equivalent screening experiment in human ESC-derived neurons would cost approximately $\$ 2,500$ to examine cell death in assays of oxygen-glucose deprivation and oxidative stress. We would expect this cost to fall significantly as high-throughput methodologies are developed. Moreover, as only drugs shown to be effective in these human systems would then be taken further into preclinical animal experiments, animal usage would be reduced. This has both ethical and financial benefits [24].

Cells derived from ESCs also have the potential to be grown either as pure cultures, to study drug effects on individual cell types or as coculture models able to examine the impact of cellular interactions. For example, it is already possible to create a microfluidic model of the blood-brain barrier [25]. This could be populated by human stem cellderived endothelial cells, astrocytes and neurons to permit study of the damaging effects of hyperglycaemia on bloodbrain barrier integrity, drugs that modify endothelial tight junction function or the dynamics of reperfusion injury after stroke. Similarly, lithographic preparation of sophisticated three-dimensional scaffolds already allows the study of interactions between cultured human neurons and Schwann cells [26].

Recent advances in stem cell biology provide the means to generate functionally relevant differentiated human neuronal and glial cell lines. These preparations are practical because exactly the same cells can be produced time after time and the desired cells can be produced in sufficient numbers to permit high-throughput screening during the early stages of drug development. High-throughput microscopy and functional assessment now also offer highly reproducible tools to examine drug-induced alterations of phenotype in these testing systems. Importantly, as culture systems and tissue engineering evolve, we will be able to apply increasingly realistic models of stroke. While embryonic stem cells will offer the possibility of cross-laboratory consistency, inducible pluripotent stem cells from subjects with specific genetic backgrounds and genetic manipulation will eventually offer the stroke researcher many of the advantages of transgenic animals in probing stroke biology.

The cost of stroke to the community is large but so is the cost of drug development, and recurrent translational failure reduces the likelihood of future research and development investment. We do not propose that interventions which are effective in an in vitro screening system should normally be taken directly to clinical trial. Nor do we suggest that effective stroke drugs will only be found for targets that are unique to humans. Rather, we suggest that human tissues can now effectively contribute to our screening armamentarium to ensure that we do not waste effort on agents with little chance of success in humans.
Which molecules should be screened for activity? If, as the literature suggests, the quality of in vivo testing has been poor, it would seem sensible to re-screen the agents previously reported as having activity in animal in vivo and in vitro experiments [1]. Before progressing to the millions of new chemical entities in pharmaceutical company libraries, it would also seem logical to screen the approximately 22,000 drugs already approved by FDA for other indications.

We use model systems to reduce complexity to manageable levels and allow us to more rapidly indentify interesting science, in this case drugs to treat human disease. However, once good candidates have been identified, complexity must be reintroduced to make sure that unforeseen interactions within the body do not negate the desired therapeutic effect. To this end, we believe that in vitro human screening will be more cost-effective than taking every agent straight into whole animal experiments. The majority of those agents which show interesting biology in vitro should then progress through rigorous experiments in small laboratory animals where the key facets of stroke systems biology such as time frame of effects, penumbral salvage, influence of co-morbidities and influence of co-treatments can be probed to ensure that the candidate drugs' effects are consistent with our understanding of stroke biology. The experience with TGN1412 indicates that we cannot assume that non-human primate testing will provide adequate confirmation of activity/safety before progressing to a clinical trial. Moreover, we cannot escape the possibility that some drugs might only work in humans. Only testing in human tissues will identify such drugs, but the onward path to clinical trial will involve more risk than normal as whole animal data would be absent. One solution to this might be to require confirmation of biological targeting and effect with tools such as positron emission tomography which uses tracer quantities of the drug before looking for therapeutic effects. We believe that stem cell-derived human culture systems will make the road to the clinic less rocky.

Sources of Funding This work was supported by an NHMRC grant and State Government Funding.

Conflict of Interest None of the authors have a competing interest to declare.

\section{References}

1. O'Collins VE, Macleod MR, Donnan GA, Horky LL, van der Worp BH, Howells DW. 1,026 Experimental treatments in acute stroke. Am Neurol Assoc. 2006;59:467-77.

2. Schulz KF, Altman DG, Moher D, for the CG. CONSORT 2010 Statement: updated guidelines for reporting parallel group randomised trials. PLoS Med. 2010;7(3):e1000251. doi:10.1371/ journal.pmed.1000251. 
3. Sena E, van der Worp HB, Howells D, Macleod M. How can we improve the pre-clinical development of drugs for stroke? Trends Neurosci. 2007;30(9):433-9.

4. Howells DW, Porritt MJ, Rewell SSJ, O'Collins V, Sena ES, van der Worp HB, et al. Different strokes for different folks: the rich diversity of animal models of focal cerebral ischemia. J Cereb Blood Flow Metab. 2010;30(8):1412-31.

5. van der Worp HB, Macleod MR. Preclinical studies of human disease: time to take methodological quality seriously. J Mol Cell Cardiol. 2011;51(4):449-50. doi:10.1016/j.yjmcc.2011.04.008.

6. Li WH. Molecular evolution. Sunderland: Sinauer; 1997.

7. Ho PW, Reutens DC, Phan TG, Wright PM, Markus R, Indra I, et al. Is white matter involved in patients entered into typical trials of neuroprotection? Stroke. 2005;36(12):2742-4.

8. DeFelipe J, Alonso-Nanclares L, Arellano JI. Microstructure of the neocortex: comparative aspects. J Neurocytol. 2002;31(3):299316.

9. Zhao S, Shetty J, Hou L, Delcher A, Zhu B, Osoegawa K, et al. Human, mouse and rat genome large-scale rearrangements: stability versus speciation. Genome Res. 2004;14:1851-60.

10. Gibbs RA, Rogers J, Katze MG, Bumgarner R, Weinstock GM, Mardis ER, et al. Evolutionary and biomedical insights from the rhesus macaque genome. Science. 2007;316(5822):222-34.

11. Thomas H. Lessons from TGN1412. Lancet. 2006;368 (9547):1569-70. doi:10.1016/s0140-6736(06)69651-7.

12. Barnes MR, Holbrook J, Feild JA, Searls DB, Sanseau P. Risk in drug trials. Lancet. 2006;368(9554):2205.

13. Dávalos A, Castillo J, Serena J, Noya M. Duration of glutamate release after acute ischemic stroke. Stroke. 1997;28(4):708-10.

14. Williams SM, Sullivan RKP, Scott HL, Finkelstein DI, Colditz PB, Lingwood BE, et al. Glial glutamate transporter expression patterns in brains from multiple mammalian species. Glia. 2005;49 (4):520-41. doi:10.1002/glia.20139.

15. Pouton CW, Haynes JM. Embryonic stem cells as a source of models for drug discovery. Nat Rev Drug Discov. 2007;6 (8):605-16.
16. Marcoli M, Bonfanti A, Roccatagliata P, Chiaramonte G, Ongini E, Raiteri $\mathrm{M}$, et al. Glutamate efflux from human cerebrocortical slices during ischemia: vesicular-like mode of glutamate release and sensitivity to A2A adenosine receptor blockade. Neuropharmacology. 2004;47(6):884-91.

17. Werth JL, Park TS, Silbergeld DL, Rothman SM. Excitotoxic swelling occurs in oxygen and glucose deprived human cortical slices. Brain Res. 1998;782(1-2):248-54.

18. Römer PS, Berr S, Avota E, Na S-Y, Battaglia M, ten Berge I, et al. Preculture of PBMCs at high cell density increases sensitivity of T-cell responses, revealing cytokine release by $\mathrm{CD} 28$ superagonist TGN1412. Blood. 2011;118(26):6772-82. doi:10.1182/blood-201012-319780.

19. Kaufman DS, Hanson ET, Lewis RL, Auerbach R. Hematopoietic colony-forming cells derived from human embryonic stem cells. Proc Natl Acad Sci USA. 2001;98(19):1071-721.

20. The National Institutes of Health resource for stem cell research. Stem cell information http://stemcells.nih.gov. In: Stem cell information USA. 2012.

21. Bock C, Kiskinis E, Verstappen G, Gu H, Boulting G, Smith ZD, et al. Reference maps of human ES and iPS cell variation enable high-throughput characterization of pluripotent cell lines. Cell. 2011;144(3):439-52. doi:10.1016/j.cell.2010.12.032.

22. Goldberg MP, Strasseer U, Dugan L. Techniques for assessing neuroprotective drugs in vitro. Int Rev Neurobiol. 1997;40:69-93.

23. Green AR, Cross AJ. Neuroprotective agents and cerebral ischaemia. London: Academic; 1997. London.

24. Howells DW, Sena ES, O'Collins V, Macleod MR. Improving the efficiency of the development of drugs for stroke. Int J Stroke. 2012;7(5):371-7.

25. Booth R, Kim H. Characterization of a microfluidic in vitro model of the blood-brain barrier ( $\mu$ BBB). Lab Chip. 2012;12(10):1784-92.

26. Koroleva A, Gill AA, Ortega I, Haycock JW, Schlie S, Gittard SD, et al. Two-photon polymerisation-generated and micromoldingreplicated 3D scaffolds for peripheral neural tissue engineering applications. Biofabrication. 2012;4(2):025005. 